\title{
Развитие взглядов на взаимосвязь произвольного движения и его мысленного образа
}

\section{Игорь В. Каминский", Сергей В. Леонов}

Московский государственный университет им. М. В. Ломоносова, г. Москва, Российская Федерация

*E-mail: min5drav@mail.ru

\begin{abstract}
Аннотация
Ввеление. Широко известно, что проработка Авигательного навыка посреАством мысленного преАставления способствует повышению его скоорАинированности и результативности. ОАнако образ рассматривался как гипотетическая основа произвольного Авижения еще Ао начала своего практического применения. Статья впервые представляет текущее понимание образа движения как разрешения противоречий, присущих более ранним взглядам, и рассматривает совместимость послеАних с современной точкой зрения, чем обосновывает необхолимость пересмотра некоторых существующих рекомендаций по применению мысленного образа в спорте.
\end{abstract}

Теоретическое обоснование. Отправной точкой в рассмотрении мысленного образа как основы произвольного Авижения яви^ась илеомоторная теория. Моторным эфрфектам, описанным как неконтролируемое выражение Аоминирующей илеи, посвящены работы Т. Аэйкока и У.Б. Карпентера. ОАнако их видение как прояв^ения системной роли образа в контроле Авижения приш^о благодаря трудам И.Ф. Гербарта, Г.Р. Аотце и Э. Харлесса. Экспериментальное подтвержАение подобных явлений было получено в ряде инструментальных исслеАований непроизвольной Авигательной активности при ее мысленном преАставлении, которые подАержали научный интерес к иАеомоторной теории в условиях ее критики со стороны бихевиоризма. Разрешение противоречий было предложено на современном этапе М. Ажиннеродом, рассматривающим Авигательный образ как осознанную репрезентацию лежащих в основе соответствующего Авижения нейрофизиологических процессов, которая фрормируется при отсутствии инактивирующей обратной связи от исполнительных органов.

Результаты и их обсужАение. Современное понимание Авигательного образа фокусируется на его эквивалентности фактическому Авижению с точки зрения реализующих его центральных нейрофризиологических процессов и присущих ему свойств, в т. ч. фрормирования навыка при повторении соответствующего Авижения. Важную роль в Авигательном обучении, особенно на начальных этапах, которую подчеркивал еще П.Ф. Аесгафт, играют когнитивный анализ 


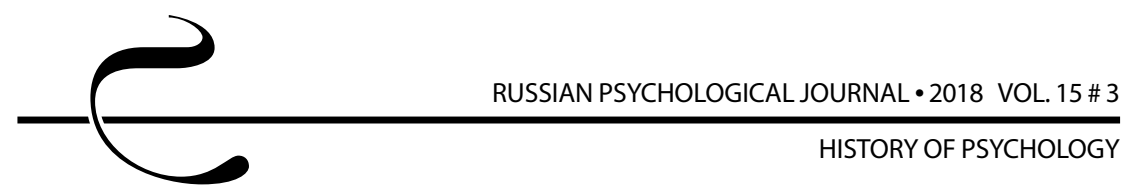

Авижения и познание его смысловой структуры с помощью образа. При этом, как показывают исследования, когнитивные и моторные процессы тесно переплетены межАу собой.

\section{КАючевые слова}

психология спорта, история психологии, мысленный образ, идеомоторный эфрфрект, илеомоторная теория, Авигательный навык, Авигательное умение, произвольное Авижение, мысленная проработка, мысленная тренировка

\section{Основные положения}

- на протяжении Авух веков мысленный образ рассматривается как основа произвольного Авижения: Аанная илея является кАючевой в рамках теории илеомоторного контроля Авижения;

- классическая илеомоторная теория гласит, что произвольное Авижение контролируется на основе отражающих его результат ощущений, испытанных при прелшествующем опыте его выполнения и представленных субъекту в виле образа; если образ Авижения Аостаточно яркий, он может повлечь за собой еАва заметный Авигательный ответ, который, в свою очередь, будет увеличивать реалистичность вызвавшего его образа посреАством обратной связи;

- разрешением противоречий в иАее контроля произвольного Авижения на основе его образа, на которые указывалось еще с начала XX в., стало признание изменчивости Авигательного образа и его роли в когнитивном анализе осваиваемых Аействий, а также предположение об инактивации моторных репрезентаций еще АО их осознания поА влиянием обратной связи, сигнализирующей о выполнении $\triangle$ Аижения;

- на современном этапе Авигательный образ рассматривается как нейросризио^огический эквивалент реального Авижения, исполнение которого тормозится на определенном уровне ЦНС; считается, что моторные функции играют неотьем^емую роль в реализации когнитивных процессов.

\section{Для цитирования}

Каминский И. В., Леонов С.В. Развитие взглядов на взаимосвязь произвольного движения и его мысленного образа // Российский психологический журнал. 2018. Т. 15, № 3. C. 8-24. DOI: 10.21702/rpj.2018.3.1

Материалы статьи получены 06.11.2017 


\title{
Evolution of Views on the Relationship Between Voluntary Movements and Their Mental Images
}

\author{
Igor V. Kaminskiy*, Sergey V. Leonov
}

Lomonosov Moscow State University, Moscow, Russian Federation

*Corresponding author: $\underline{\text { min5drav@mail.ru }}$

\begin{abstract}
Introduction. Mental rehearsal of motor skill performance improves its coordination and effectiveness. However, mental image has been considered to be a hypothetical basis for voluntary movements even before its practical use begins. The present paper provides an original view on the current motor imagery understanding as a resolution of conflicts inherent to earlier concepts. The compatibility between historical and modern motor imagery theories is discussed. Thereby, revision of some of existing recommendations concerning mental imagery use in sports is underpinned.
\end{abstract}

Theoretical Basis. Ideomotor theory was the first concept considering mental imagery as a basis for voluntary movements. T. Laycock and W. B. Carpenter described motor consequences of uncontrolled expression of a dominant idea. In the context of H. Herbart's, R. H. Lotze's, and E. Harless' work, this phenomenon can be viewed more broadly in the light of systemic role of mental imagery in controlling movements. A number of instrumental studies of involuntary motor activity during movement imagination provided experimental confirmation of such phenomena and supported scientific interest in the ideomotor theory when it was criticized by the behaviorism. In the modern period, to resolve existing contradictions, $M$. Jeannerod has suggested considering the motor image as a conscious representation of neurophysiological processes underlying the corresponding movement, which is formed in the absence of inactivating afferent response of effector organs.

Results and Discussion. The modern understanding of the motor imagery focuses on its equivalence to the actual movement in terms of the underlying central neurophysiological processes and their functions, including formation of skills with the repetition of a corresponding movement. Following P. F. Lesgaft's idea, an individual's cognitive analysis of movements and understanding of its logical structure by means of mental imagery play an important role in motor learning, especially in its initial stages. Herewith, research shows that cognitive and motor processes are closely intertwined.

\section{Keywords}

sport psychology, history of psychology, mental image, ideomotor effect, ideomotor theory, motor skill, motor ability, voluntary movement, mental practice, mental training 


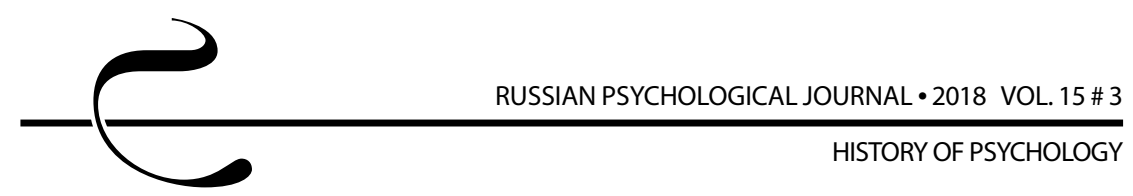

\section{Highlights}

- For two centuries, mental image has been considered a basis for voluntary movements. This is the main idea of the ideomotor theory of action control.

- The classical ideomotor theory postulates that voluntary movement is controlled by the sensations of its result, which were experienced during its previous execution and represented in the form of mental image. If the motor image is vivid enough, it can entail a barely noticeable motor response, which in turn will increase the vividness of the image via feedback.

- Since the beginning of the 20th century, it was suggested that contradictions in the idea of controlling voluntary movements on the basis of their mental images can be resolved by recognizing the dynamic nature of motor image and its role in the cognitive analysis of motor actions to be mastered and also by assuming that the motor representations can be inactivated even before the actor becomes aware of them through the feedback that signals about the action execution.

- Currently, the motor image is considered to be a neurophysiological equivalent of a real movement with execution inhibited at a certain level of the central nervous system. Motor functions play an essential role in cognitive processes.

\section{For citation}

Kaminskiy I.V., Leonov S.V. Evolution of Views on the Relationship Between Voluntary Movements and Their Mental Images. Rossiiskii psikhologicheskii zhurnal - Russian Psychological Journal, 2018, V. 15, no. 3, pp. 8-24 (in Russian). DOI: 10.21702/rpj.2018.3.1

Original manuscript received 06.11.2017

\section{Введение}

В настоящее время мысленный образ движения широко применяется в качестве вспомогательного средства, способствующего росту скоординированности и результативности двигательных действий, тем самым ускоряя достижение целевых показателей в различных сферах деятельности, таких как спортивная подготовка, нейрореабилитация, обучение хирургическим манипуляциям и игре на музыкальных инструментах. Помимо важного прикладного значения, систематическое исследование которого ведет свою историю с 30-х - 40-х гг XX в., мысленный образ характеризуется центральным положением в целом ряде теорий организации двигательных действий и поведения, которые, в свою очередь, задают особенности методологического подхода к его применению на практике. Поэтому, учитывая актуальность вопроса, как с теоретической, так и с прикладной точки зрения, мы ставим перед собой следующие взаимосвязанные задачи: 
1. Рассмотреть исторический ход развития взглядов на взаимосвязь произвольного движения с его мысленным образом.

2. Раскрыть различные подходы к пониманию природы и функций мысленного образа движения.

3. Охарактеризовать понимание мысленного образа движения с позиций современной науки.

Решая поставленные задачи, мы сохраняем особый фокус на значении мысленного образа в спортивной подготовке.

\section{Теоретическое обоснование}

\section{Идеомоторная теория}

Роль мысленного образа как основы произвольного движения впервые подчеркивается идеомоторной теорией (от греч. «іdea» - идея и лат. «motor» приводящий в движение). Согласно ее основному принципу, произвольное движение выстраивается на базе образа тех ощущений, которые оно приносит.

Автор термина «идеомоторный», английский естествоиспытатель У. Б. Карпентер [1], в своих работах не касался контроля произвольных движений, но всё же постулировал тесную взаимосвязь психической и моторной сфер. Он предполагал о существовании 3-х групп рефлексов, отличных по уровню замыкания рефлекторной дуги: «эксайтомоторные»опосредованная спинным мозгом двигательная реакция в ответ на рецепцию неосознаваемого раздражения (например, рвотный рефлекс при раздражении слизистой желудка); «сенсомоторные» - замыкаемый на уровне сенсорных ганглиев ответ на ощущаемый раздражитель (например, вздрагивание при неожиданном резком шуме); «идеомоторные» (категория, введенная У. Б. Карпентером) - непосредственное отражение принадлежащей высшим отделам головного мозга идеи в действии. По мнению У. Б. Карпентера [1], идеомоторный рефлекс возникает при подавлении воли как средства контроля над произвольным действием или при высоком уровне выжидающего внимания, сосредоточенного на предвкушаемом результате действия. Последнее он использует как научное объяснение ряду распространенных оккультных практик, таких как спиритизм, лозоходство и др., которое и преследовала его теория [1].

У. Б. Карпентер опирался на более ранние заключения врача Т. Лэйкока [2, 3], который наблюдал больных бешенством. Как известно, заболевание характеризуется типичными спазмами глоточной мускулатуры и удушьем при контакте с водой (т. н. «водобоязнь»). Анализируя поведение пациентов, Т. Лэйкок заметил, что моторные приступы могут быть спровоцированы не только непосредственным воздействием раздражителя, но также и его видом или даже мыслью о нем. Так происходило в ответ на предложение 
сделать глоток воды, ее косвенное упоминание в разговоре и даже на доносящийся шум льющейся жидкости.

Более широкая трактовка взаимосвязи психической и моторной сфер разрабатывалась в рамках немецкой научной школы. Так, И.Ф. Гербарт $[4,5]$ первым формулирует базовый принцип идеомоторного управления действием, постулирующий, что двигательный контроль есть стремление воспроизвести отражаемое в уме ощущение, сопряженное с желаемым действием и сохраненное во время предшествующего опыта его выполнения. По мнению И.Ф. Гербарта [5], сразу после рождения двигательная активность человека или животного инициируется спонтанно, не реализуя какого-либо намерения. Однако каждое движение порождает обратную связь в форме чувства, отражающего произошедшие с телом изменения, в процессе чего формируются устойчивые связи между полученным сенсорным результатом и двигательной программой, его обусловившей. При возникновении в дальнейшем желания повторить испытанные ранее изменения полученное ощущение «воспроизводит само себя» через связанную с ним двигательную программу [5, р. 464], при этом каждое такое повторение укрепляет однажды установленную связь между двигательным звеном и сенсорным эталоном и облегчает последующее воспроизведение соответствующего действия [5].

Как и И.Ф. Гербарт, его последователь Г.Р. Лотце [6] говорит о том, что появлению целенаправленного движения предшествует спонтанная двигательная активность, инициируемая без участия сознания, поскольку именно «тело учит разум», демонстрируя ему заложенные в него возможности [6, р. 289]. И как только взаимосвязь между действием и проистекающим из него чувственным опытом установлена, всякое порождаемое разумом желание вновь пережить данный опыт при малейшем внутреннем побуждении ставит тело перед неотвратимым «механическим обязательством» действовать в соответствии с намерением субъекта [6, р. 288].

Э. Харлесс [7], давший дальнейшее развитие описанным идеям, считает, что яркость мысленно переживаемого образа напрямую определяется силой реализующих его на психофизиологическом уровне нервных импульсов, и, чтобы произвести соответствующий двигательный ответ, вновь сформированный образ движения должен обладать яркостью, достаточной для превышения порога активации моторного центра. Однако по мере многочисленных повторений движения происходит проторение нервного пути, закрепляющее связь между сенсорной и моторной зонами, что выражается в снижении порога активации последней. Именно в этом Э. Харлесс [7] видит механизм обучения двигательным навыкам в процессе тренировки. Другим следствием закрепления взаимосвязи между движением и инициирующим его образом, согласно Э. Харлессу [7], является непроизвольная активация 
моторного центра при достаточно ярком мысленном представлении движения. Но в норме она не может быть достаточно сильна, чтобы перерастать в полноценное действие. Вместо этого действие протекает в «микромасштабах», выражаясь в едва заметных и чаще всего субъективно неразличимых двигательных актах, существующих как результат микросокращений мышц, ответственных за действительную реализацию представленного мысленно движения (ср. [1]). При этом, несмотря на отсутствие фактически выполненного движения, построенное на его основе «микродвижение» уже является достаточным для формирования обратной афферентации в сенсорные отделы мозга, которая подкрепляет переживаемый образ, усиливая его яркость [7].

\section{Инструментальные исследования периферической активности}

С приходом и становлением бихевиоризма как одного из ведущих психологических направлений научный интерес к дальнейшей разработке идеомоторной концепции временно падает. В условиях активной критики единственным фактором, объективно подтверждающим идеомоторные феномены, становятся результаты инструментальных исследований.

Так, с помощью электромиографического (ЭМГ) измерения Р. Аллерс и Ф. Щемински [8], проинструктировавшие испытуемых представить сжатие в кулак правой руки или произнести вслух «сейчас я сожму свой кулак», в $80 \%$ случаев смогли зарегистрировать биоэлектрические потенциалы в мышцах руки, которой выполнялось воображаемое движение [8]. Согласно Э. Якобсону [9], общие ЭМГ-характеристики мышц на фоне представленного движения соответствуют таковым для аналогичного реально выполненного движения (в т. ч. по локализации), однако значительно уступают им по выраженности. У.А. Шоу [10] показал, что величина биоэлектрических потенциалов, регистрируемых в мышцах на фоне представленных движений, прямо пропорциональна нагрузке, указанной при инструктировании испытуемого, которая изменяет мысленно прилагаемое усилие. Наконец, рядом исследований $[11,12]$ установлено, что временной паттерн мышечных сокращений на фоне мысленного движения воспроизводит реальные временные характеристики деятельности мышц, необходимой для его осуществления.

\section{Идеомоторная теория: критика}

Как известно, идеомоторная теория изначально выводилась только лишь на основе интроспекции и логических умозаключений и на протяжении длительного времени не имела никакого эмпирического подтверждения, что делало ее особенно подверженной критике.

Так, критикуя идеомоторную теорию, обобщенную в «Принципах психологии» У. Джемса [13], Э.Л. Торндайк [14] утверждал, что истинная причина, 


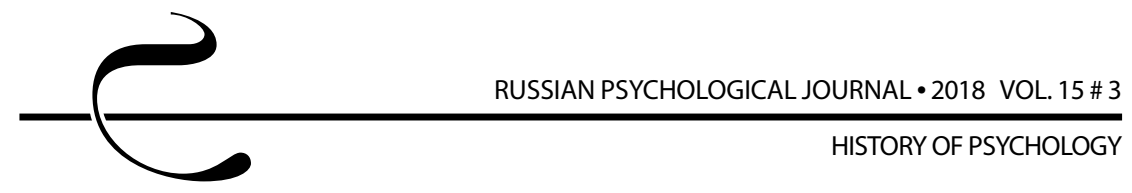

а значит и пусковой фактор действия, содержится во внешнем контексте его осуществления. Для демонстрации несостоятельности идеомоторного принципа Э.Л. Торндайк [14] попросил присутствующих на XX ежегодном собрании Американской психологической ассоциации в 1911 г. упорядочить в соответствии со степенью правдоподобности от 4-х до 10-ти утверждений, определенным образом соотносящихся с известными положениями теории идеомоторного контроля. Среди 40 утверждений, отобранных им для эксперимента, встречались оригинальные выдержки из текста «Принципов психологии» У. Джемса [13], специально сконструированные суждения, а также суждения, основанные на суевериях. Результаты проведенного ранжирования показали, что различия в правдоподобности между описаниями идеомоторного принципа и суждениями, заведомо выстроенными на предрассудках, не столь существенны [14].

С более глубокой критикой в адрес не столько ключевой идеи идеомоторного принципа, сколько развития его авторами представлений о механизмах двигательного обучения обращается Н. А. Бернштейн [15]. Рассматривая теории проторения нервного пути, Н. А. Бернштейн замечает: их сторонники по какой-то причине упускают из вида, что «человек принимается за разучивание тех или иных движений именно потому, что не умеет их делать», и «поэтому в начале разучивания навыка ему, в сущности, нечего проторять или же проторяться и запечатлеваться у него начнут те самые неловкие и неправильные движения, какие он единственно в состоянии совершить в начале работы над навыком» [15, с. 215]. Однако внутреннее логическое противоречие в концепции проторения, на которое указывает Н.А. Бернштейн [15], - судя по всему, лишь видимость, созданная базовыми отличиями взглядов представителей данного подхода на «препятствия», стоящие на пути освоения двигательного навыка. Так, Э. Харлесс [7] и другие сторонники проторения, очевидно, считали, что приступая к выполнению действия, субъект априори уже обладает четким представлением о том, что он в идеале намерен получить, но не может реализовать такое представление на практике, поскольку связь последнего с исполнительным моторным звеном еще слишком слаба для этого. Н.А. Бернштейн же видел принцип двигательного обучения не в сокращении потерь при передаче информационного сигнала, которое сводилось у Э. Харлесса к количественному нарастанию его интенсивности на периферии, а в его качественных изменениях, обусловленных идущей по мере овладевания навыком перестройкой центральных процессов - динамическим характером той идеальной модели, в соответствии с которой выстраивается фактически выполненное движение.

В этой связи выявляется и еще один существенный недостаток классической идеомоторной теории $[4,5,7]$. Если освоение навыка обусловливается 
проторением нервного пути, то роль субъекта в успешности овладевания им сводится лишь к изъявлению воли к механическому повторению действия, а легкость и ловкость, нарабатываемые в его исполнении, - неизбежное следствие упорства и стойкости занимающегося. Это исключает всякое влияние на результат со стороны качественных изменений в структуре обучающего процесса, таких как варьирование средств и методов, применяемых в нем, которое уже невозможно отрицать на современном этапе. В противовес этой идее П.Ф. Лесгафт [16] одним из первых подчеркивает важность практического аспекта создания правильного образа разучиваемого движения и благотворное влияние такого образа на процесс овладевания проблемным двигательным навыком. В результате закрепляется точка зрения, согласно которой представление о двигательном действии является не чем-то изначально данным, а напротив, - чем-то, что необходимо приобрести в процессе обучения. По П.Ф. Лесгафту, образ хоть и отражает эталон выполняемого двигательного действия, в то же время является средством активного поиска такого эталонного варианта - инструментом познания, возможностью моделирования своих действий с тем, чтобы предугадать их результат.

Необходимо отметить, что, несмотря на методологическое несовершенство разработки идеомоторной теории на ранних этапах, наиболее активные попытки опровергнуть ее (в частности, предпринятые Э.Л. Торндайком [14] и его современниками), также были построены на весьма шаткой методологии. Показательными в этом плане могут быть работы Р. Вудвортса $[17,18]$, вступающие в прямое противоречие с положениями об идеомоторном контроле У. Джемса [13]. Так, согласно У. Джемсу [13], произвольному движению всегда предшествует идея или мысленный образ такого движения. В свою очередь, Р. Вудвортс [17] в эксперименте с привлечением хорошо обученных интроспекции участников демонстрирует, что лишь малая часть полученных им сообщений указывает на соответствие между движением и содержанием переживаемого непосредственно перед его выполнением мысленного образа, тогда как примерно в половине отчетов вовсе никакое образное представление не предшествует двигательной активности. Несколько ранее Р. Вудвортс [18] описывает свои попытки выполнить сгибание и разгибание большого пальца независимо от остальных пальцев ноги. Согласно наблюдениям автора, его усилия, направленные на то, чтобы блокировать движение 4-х пальцев, представились надежным способом заставить их двигаться. Принимая во внимание результаты Дж.Х. Баира [19], также свидетельствующие о неэффективности препятствующих идей в предотвращении движений, Р. Вудвортс [17] оспаривает второе положение У. Джемса, гласящее, что в случаях, когда образ не трансформируется в соответствующее ему действие, «на кромке 


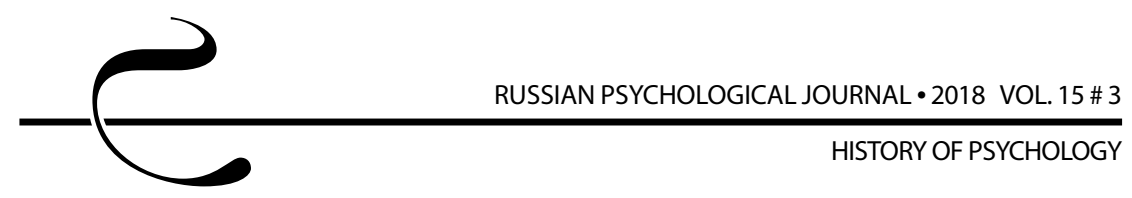

сознания» можно обнаружить противодействующую идею, блокирующую исходное намерение [8, р. 525-527].

\section{Разрешение противоречий}

Пусть последние приведенные наблюдения и были проведены с помощью методов интроспекции, имеющих известную долю субъективизма, сторонники идеомоторного принципа долгое время не располагали аргументами, позволяющими объяснить выявленные противоречия. Данная задача была решена лишь в 1994 г. в теории, предложенной французским нейрофизиологом М. Джиннеродом [20], который выделил в качестве отдельных конструктов образ движения и моторную репрезентацию. Согласно М. Джиннероду [20], и образ движения, и моторная репрезентация несут в себе информацию, позволяющую точно задать соответствующее двигательное действие: его временные рамки, двигательный состав, необходимые силовые затраты, пространственную конфигурацию и требуемый итоговый результат. Однако, в отличие от мысленного образа, моторная репрезентация возникает на стадии подготовки двигательного действия и существует чрезвычайно короткое время, поскольку инактивируется поступающей от исполнительных органов афферентацией, свидетельствующей о выполнении соответствующего действия.

В результате, ввиду ограничения сознательных процессов по скорости их протекания при успешном выполнении двигательного действия, моторная репрезентация блокируется раньше, чем может быть осознана субъектом, вследствие чего выполнение действия не сопровождается переживанием его мысленного образа. Однако если инактивирующая афферентация не поступает по какой-либо причине, активная моторная репрезентация становится доступной сознанию субъекта, за счет чего трансформируется в образ движения, который, согласно М. Джиннероду [20], является осознанной моторной репрезентацией. Так, есть данные, что при неспособности выполнения определенных движений на фоне интактности мозговых структур, обеспечивающих их программирование, в частности, при ампутации [21] или деафферентации [22] конечностей, а также при повреждениях спинного мозга [23], субъекты формируют ясный образ намеченного действия, когда перед ними стоит задача его осуществления. Наконец, в случае осознанного целенаправленного представления двигательного действия, активность исполнительных органов (а значит, и инактивирующая афферентация) блокируется автоматически, что определяет произвольность такого представления [20].

\section{Результаты и их обсуждение}

Обобщая приведенные данные, можно выделить два подхода к пониманию природы мысленного образа движения (см. [24]). В рамках первого, 
разрабатываемого еще в классической идеомоторной теории $[4,5,6,7]$, мысленный образ движения рассматривается как моторная репрезентация, содержащая исчерпывающую информацию, которая специфицирует соответствующее движение и является его пусковым фактором. Второй подход явился крайней реакцией на чрезмерную механистичность классической идеомоторной теории и необходимость утверждения мысленного образа как инструмента познания. В рамках такого подхода мысленный образ считается когнитивным планом, отражающим только временную последовательность и смысловой состав необходимых действий, тогда как информация об их механокинематических характеристиках не выходит за пределы обособленного исполнительного звена и поэтому не представлена сознанию. В результате образ выступает как когнитивная репрезентация индивидуальных целей и намерения действовать и является посредником между волей субъекта и ее исполнением [25].

Отождествление образа движения с моторной репрезентацией подразумевает также и аналогию их свойств, в т. ч. сопряженность с процессами, отвечающими за преобразование моторной программы и совершенствование навыка. Предполагалось, что основой такого процесса может быть микродвигательная активность, дающая обратную связь, которая способствует формированию сенсорных коррекций подобно тому, как это происходит при выполнении полноценного движения [26]. Однако на современном этапе гипотеза о ведущей роли афферентации в мысленной проработке двигательных навыков теряет свою актуальность, поскольку в ходе специальных исследований подобие паттернов мышечной активности, разворачивающейся в процессе реального выполнения действия и его мысленного представления, выявлено не было [27]. Напротив, к настоящему времени масса экспериментальных данных свидетельствует о центральной локализации процессов, способствующих развитию моторного навыка за счет его мысленного повторения [24].

Если рассматривать мысленный образ как смысловое отражение действия, изолированное от его механических характеристик, понимание механизмов, опосредующих его влияние на моторные функции, существенно изменяется. Как когнитивный инструмент образ может обеспечивать анализ действия, вычленение последовательности отдельных операций, их закрепление в памяти и акцентирование в ходе дальнейшей проработки, поиск оптимальной стратегии достижения поставленной двигательной задачи [28].

С позиций современной науки, подходы к мысленному образу как к моторной репрезентации и как к средству познания не только не противоречат друг другу, но и формируют синтез целостного понимания образа в свете последних данных об участии моторных программ в когнитивных 
процессах (см. [24]). Однако в случае со сложнокоординационными навыками считается, что соотношение когнитивных и моторных механизмов в их репрезентации может быть разным в зависимости от уровня владения ими: вырабатывание навыка начинается с усвоения его смысловой структуры, и лишь в процессе дальнейшей фактической проработки формируется моторная репрезентация [29].

\section{Заключение}

Проведенный анализ литературы иллюстрирует современные взгляды на природу и свойства мысленного образа движения как результат двухвекового пути обогащения и совершенствования представлений об образе как об основе произвольного движения. Понимание предпосылок современного видения проблемы дает возможность целостного восприятия концепта мысленного образа движения, не ограниченного рамками какого-либо из подходов, четко определяя их взаимные различия, и в то же время помогает критически осмыслить некоторые из них, принимая во внимание их известные недостатки. Так, может считаться бесспорной роль мысленного образа как средства разработки плана действий, закрепления их последовательности, мысленного предвосхищения результата. Однако отрицание совершенствования двигательных умений и навыков посредством активации моторной системы при переживании образа также немыслимо с учетом многочисленных нейровизуализационных данных об эквивалентности действия и его умозрительного повторения и даже вовлеченности моторных программ в когнитивную деятельность.

Хочется также подчеркнуть практическую значимость представленной работы как «путеводителя», призванного обращать внимание авторов и практикующих специалистов на корректность методологической основы планируемых или интерпретируемых исследований и прикладных методик, применяемых в условиях реальной деятельности. С учетом современных представлений об образе как об эквиваленте реального действия, тормозящемся на определенном уровне ЦНС, научно обоснованный подход заключается в достижении максимального соответствия содержания мысленного образа условиям, в которых фактически разворачивается отрабатываемая активность [30]. В то же время весьма распространенными сейчас являются рекомендации, акцентирующие внимание субъектов на активности мышц, сопровождающей процесс мысленного представления движений.

Постулируя, что реалистично представленная мышечная работа на грани с ее фактической активизацией является одним из критериев достижения цели представления, подобные рекомендации имеют расчет на запуск изменений в центральной моторной программе по механизмам обратной 
связи с периферии, что отражает принципы классической идеомоторной теории. Однако в соответствии с современными взглядами, разрешившими более ранние концептуальные противоречия, восходящая афферентация, напротив, выполняет роль деактиватора моторной программы. Более того, если отталкиваться от эквивалентности процессов, лежащих в основе образа и реального действия, концентрация внимания на двигательном звене во время представления может иметь пагубное влияние на последующую автоматичность исполнения моторного навыка [15]. Таким образом, по результатам проделанной работы можно обозначить основания для пересмотра некоторых существующих рекомендаций по применению мысленного образа в спорте.

\section{Благодарности}

Работа выполнена при поддержке гранта РФФИ № 18-013-01211.

\section{Acknowledgments}

Supported by the Russian Foundation for Basic Research grant no. 18-013-01211.

\section{Литература}

1. Carpenter W. B. On the Influence of Suggestion in Modifying and directing Muscular Movement, independently of Volition // Proceedings of the Royal Institution. 1852. 1.P. 147-154. URL: https://ia601305.us.archive.org/5/items/ b22377074/b22377074.pdf (Accessed 20.08.2018).

2. Laycock T. A treatise on the nervous diseases of women; comprising an inquiry into the nature, causes, and treatment of spinal and hysterical disorders. London: Longmans, 1840. 402 p.

3. Laycock T. On the reflex functions of the brain // Reprinted from N. XXXVII of The British and Foreign Medical Review. Bartholomew Close, UK: Adlard, 1845. P. 1-16.

4. Herbart J. F. Lehrbuch zur Psychologie. Königsberg und Liepzig: Bey August Wilhelm Unzer, 1816. 214 p.

5. Herbart J. F. Psychologie als Wissenschaft neu gegründet auf Erfahrung, Metaphysik und Mathematik. Analytischer Teil. Verf., 1825. 541 p.

6. Lotze R. H. Medizinische Psychologie oder Physiologie der Seele. Leipzig, Germany: Weidmann'sche Buchhandlung, 1852.

7. Harless E. Der Apparat des Willens // I. H. Fichte, H. Ulrici, I. U. Wirth (Eds.) Zeitschrift fur Philosophie und philosophische Kritik. Vol. 38. Halle, Germany: Pfeffer, 1861. P. 50-73.

8. Allers R., Scheminzky F. über Aktionsströme der Muskeln bei motorischen Vorstellungen und verwandten Vorgängen // Pflüger's Archiv für die gesamte 
Physiologie des Menschen und derTiere (Pflügers Archiv - European Journal of Physiology). 1926. Vol. 212, Issue 1.P. 169-182. DOI: $10.1007 / B F 01723129$

9. Jacobson E. Electrical Measurements of Neuromuscular States During Mental Activities (Part I). Imagination of Movement Involving Skeletal Muscle // American Journal of Physiology. 1930. Vol. 91, Issue 2. P. 567-608. DOI: 10.1152/ajplegacy.1930.91.2.567

10. Shaw $W$. A. The relation of muscular action potentials to imaginal weight lifting // Archives of Psychology. 1940. № 247. P. 5-50.

11. Suinn R. M. Psychology and sports performance: Principles and applications // R. M. Suinn (Ed.) Psychology in sports: Methods and applications. Minneapolis: Burgess, 1980. P. 26-36.

12. Wehner T., Vogt S., Stadler M. Task-specific EMG-characteristics during mental training // Psychological Research. 1984. Vol. 46, № 4. P. 389-401.

13. James W. Principles of Psychology. London: Macmillan, 1890.

14. Thorndike E. L. Ideo-motor action // Psychological Review. 1913. Vol. 20 (2). P. 91-106. DOI: $10.1037 / \mathrm{h} 0072027$

15. Бернштейн Н. А. О ловкости и ее развитии. М.: Физкультура и спорт, 1991. 288 c.

16. Лесгафт П. Ф. Собрание педагогических сочинений / под ред. Г. Г. Шахвердова и др. Т. 2. М.: Физкультура и спорт, 1952. 383 с.

17. Woodworth R. S. The cause of a voluntary movement // J. H. Tufts (Ed.) Studies in Philosophy and Psychology. Garman memorial volume. Boston: Houghton-Mifflin, 1906. P. 351-392.

18. Woodworth R. S. Le Mouvement // Science. 1904. Vol. 20, Issue 498. P. 78-79. DOI: $10.1126 /$ science. 20.498 .78

19. Bair J. H. Development of voluntary control // Psychological Review. 1901. 8 (5). P. 474-510. DOI: $10.1037 /$ h0074157

20. Jeannerod $M$. The representing brain: Neural correlates of motor intention and imagery // Behavioral and Brain Sciences. 1994. Vol. 17, Issue 2. P. 187-202. DOI: $10.1017 /$ S0140525X00034026

21. Schilder P. The Image and Appearance of the Human Body. London: Routledge and Kegan Paul, 1935. 353 p.

22. Jeannerod M., Michel F., Prablanc C. The control of hand movements in a case of hemianaesthesia following a parietal lesion // Brain. 1984. Vol. 107, № 3. P. 899-920.

23. Decety J., Boisson D. Effect of brain and spinal cord injuries on motor imagery // European Archives of Psychiatry and Clinical Neurosciences. 1990. Vol. 240, Issue 1. P. 39-43.

24. Каминский И. В., Веракса А. Н. Традиционные теории и современные взгляды на природу мысленного образа движения: применение в спортивной практике // Национальный психологический журнал. 2017. № 2 (26). С. 16-25. 
25. Sackett R. S. The Influence of Symbolic Rehearsal upon the Retention of a Maze Habit // The Journal of General Psychology. 1934. Vol. 10, Issue 2. P. 376-398. DOI: $10.1080 / 00221309.1934 .9917742$

26. Arnold M. B. On the mechanism of suggestion and hypnosis //The Journal of Abnormal and Social Psychology. 1946. 41 (2). P. 107-128.

27. Slade J. M., Landers D. M., Martin P. E. Muscular activity during real and imagined movements: A test of inflow explanations // Journal of Sport and Exercise Psychology. 2002. Vol. 24, Issue 2. P. 151-167.

28. Ryan E. D., Simons J. Cognitive Demand, Imagery, and Frequency of Mental Rehearsal as Factors Influencing Acquisition of Motor Skills // Journal of Sport Psychology. 1981. Vol. 3, Issue 1. P. 35-45. DOI: 10.1123/jsp.3.1.35

29. Olsson C. J., Nyberg L. Brain simulation of action may be grounded in physical experience // Neurocase. 2011. Vol. 17, Issue 6. P. 501-505. DOI: 10.1080/13554794.2010.547504

30. Holmes P. S., Collins D. J. The PETTLEP Approach to Motor Imagery: A Functional Equivalence Model for Sport Psychologists // Journal of Applied Sport Psychology. 2001. Vol. 13, Issue 1.P.60-83. DOI:10.1080/10413200109339004

\section{References}

1. Carpenter W. B. On the influence of suggestion in modifying and directing muscular movement, independently of volition. Proceedings of the Royal Institution, 1852, no. 1, pp. 147-154. Available at: https://ia601305.us.archive. org/5/items/b22377074/b22377074.pdf (Accessed 20 August 2018).

2. Laycock T. A treatise on the nervous diseases of women; comprising an inquiry into the nature, causes, and treatment of spinal and hysterical disorders. London, Longmans, 1840. $402 \mathrm{p}$.

3. LaycockT. On the reflex functions of the brain. Reprinted from N. XXXVII of The British and Foreign Medical Review, Bartholomew Close, UK, Adlard, 1845, pp. 1-16.

4. Herbart J. F. Lehrbuch zur Psychologie. Königsberg und Liepzig: Bey August Wilhelm Unzer, 1816. 214 p.

5. Herbart J. F. Psychologie als Wissenschaft neu gegründet auf Erfahrung, Metaphysik und Mathematik. Analytischer Teil. Verf., 1825. 541 p.

6. Lotze R. H. Medizinische Psychologie oder Physiologie der Seele. Leipzig, Germany, Weidmann'sche Buchhandlung, 1852.

7. Harless E. Der Apparat des Willens. In: I. H. Fichte, H. Ulrici, I. U. Wirth (eds.) Zeitschrift fur Philosophie und philosophische Kritik, V. 38. Halle, Germany, Pfeffer, 1861, pp. 50-73.

8. Allers R., Scheminzky F. über Aktionsströme der Muskeln bei motorischen Vorstellungen und verwandten Vorgängen. Pflüger's Archiv für die gesamte 
Physiologie des Menschen und der Tiere (Pflügers Archiv - European Journal of Physiology), 1926, V. 212, Issue 1, pp. 169-182. DOI: $10.1007 / B F 01723129$

9. Jacobson E. Electrical measurements of neuromuscular states during mental activities (Part I). Imagination of movement involving skeletal muscle. American Journal of Physiology, 1930, V. 91, Issue 2, pp. 567-608. DOI: 10.1152 / ajplegacy.1930.91.2.567

10. Shaw W. A. The relation of muscular action potentials to imaginal weight lifting. Archives of Psychology, 1940, no. 247, pp. 5-50.

11. Suinn R. M. Psychology and sports performance: Principles and applications. In: R. M. Suinn (ed.) Psychology in sports: Methods and applications. Minneapolis, Burgess, 1980, pp. 26-36.

12. WehnerT., Vogt S., Stadler M. Task-specific EMG-characteristics during mental training. Psychological Research, 1984, V. 46, no. 4, pp. 389-401.

13. James W. Principles of psychology. London, Macmillan, 1890.

14. Thorndike E. L. Ideo-motor action. Psychological Review, 1913, V. 20 (2), pp. 91-106. DOI: $10.1037 / \mathrm{h} 0072027$

15. Bernstein N. A. O lovkosti i ee razvitii [Dexterity and its development]. Moscow, Fizkul'tura i sport Publ., 1991. 288 p.

16. Lesgaft P. F., Shakhverdov G. G. (ed.) Sobranie pedagogicheskikh sochinenii [Collection of pedagogical works:V.2]. Moscow, Fizkul'tura i sport Publ., 1952. 383 p.

17. Woodworth R. S. The cause of a voluntary movement. In: J. H. Tufts (ed.) Studies in philosophy and psychology. Garman memorial volume. Boston, Houghton-Mifflin, 1906, pp. 351-392.

18. Woodworth R. S. Le movement. Science, 1904, V. 20, Issue 498, pp. 78-79. DOI: $10.1126 /$ science. 20.498 .78

19. Bair J. H. Development of voluntary control. Psychological Review, 1901, 8 (5), pp. 474-510. DOI: $10.1037 /$ h0074157

20. Jeannerod M. The representing brain: Neural correlates of motor intention and imagery. Behavioral and Brain Sciences, 1994, V. 17, Issue 2, pp. 187-202. DOI: $10.1017 /$ S0140525X00034026

21. Schilder P. The image and appearance of the human body. London, Routledge and Kegan Paul, 1935. 353 p.

22. Jeannerod M., Michel F., Prablanc C. The control of hand movements in a case of hemianaesthesia following a parietal lesion. Brain, 1984, V. 107, no. 3, pp. 899-920.

23. Decety J., Boisson D. Effect of brain and spinal cord injuries on motor imagery. European Archives of Psychiatry and Clinical Neurosciences, 1990, V. 240, Issue 1, pp. 39-43.

24. Kaminskii I. V., Veraksa A. N. Conventional theories and modern views on the nature of mental imaging of movements used in sport. Natsional'nyi 
psikhologicheskii zhurnal - National Psychological Journal, 2017, no. 2 (26), pp. 16-25 (in Russian).

25. Sackett R. S. The influence of symbolic rehearsal upon the retention of a maze habit. The Journal of General Psychology, 1934, V. 10, Issue 2, pp. 376-398. DOI: $10.1080 / 00221309.1934 .9917742$

26. Arnold M. B. On the mechanism of suggestion and hypnosis. The Journal of Abnormal and Social Psychology, 1946, 41 (2), pp. 107-128.

27. Slade J. M., Landers D. M., Martin P. E. Muscular activity during real and imagined movements: A test of inflow explanations. Journal of Sport and Exercise Psychology, 2002, V. 24, Issue 2, pp. 151-167.

28. Ryan E. D., Simons J. Cognitive demand, imagery, and frequency of mental rehearsal as factors influencing acquisition of motor skills. Journal of Sport Psychology, 1981, V. 3, Issue 1, pp. 35-45. DOI: 10.1123/jsp.3.1.35

29. Olsson C. J., Nyberg L. Brain simulation of action may be grounded in physical experience. Neurocase, 2011, V. 17, Issue 6, pp. 501-505. DOI: 10.1080/13554794.2010.547504

30. Holmes P. S., Collins D. J. The PETTLEP approach to motor imagery: A functional equivalence model for sport psychologists. Journal of Applied Sport Psychology, 2001, V. 13, Issue 1, pp. 60-83. DOI: 10.1080/10413200109339004 